\title{
Effect of Interfacial Interaction on the Cross- Sectional Morphology of Tobacco Mosaic Virus using GISAXS
}

\author{
Byeongdu Lee, ${ }^{a^{*}}$ Chieh-Tsung Lo, ${ }^{a}$ P. Thiyagarajan, ${ }^{b}$ Randall E. Winans, ${ }^{a}$ Xuefa Li, ${ }^{a}$ \\ Zhongwei Niu, ${ }^{c}$ Qian Wang ${ }^{c^{*}}$ \\ ${ }^{a}$ X-ray Science Division, Advanced Photon Source, Argonne National Lab., 9700 S. \\ Cass Ave., Argonne, IL 60439, USA \\ ${ }^{\mathrm{b}}$ Intense Pulsed Neutron Source, Argonne National Lab., 9700 S. Cass Ave., Argonne, IL \\ 60439, USA. \\ ${ }^{c}$ Department of Chemistry and Biochemistry and Nanocenter, University of South \\ Carolina, 631 Sumter Street, Columbia, SC 29208, USA \\ * blee@aps.anl.gov (BL), wang@mail.chem.sc.edu (QW)
}

\section{Supporting Information}




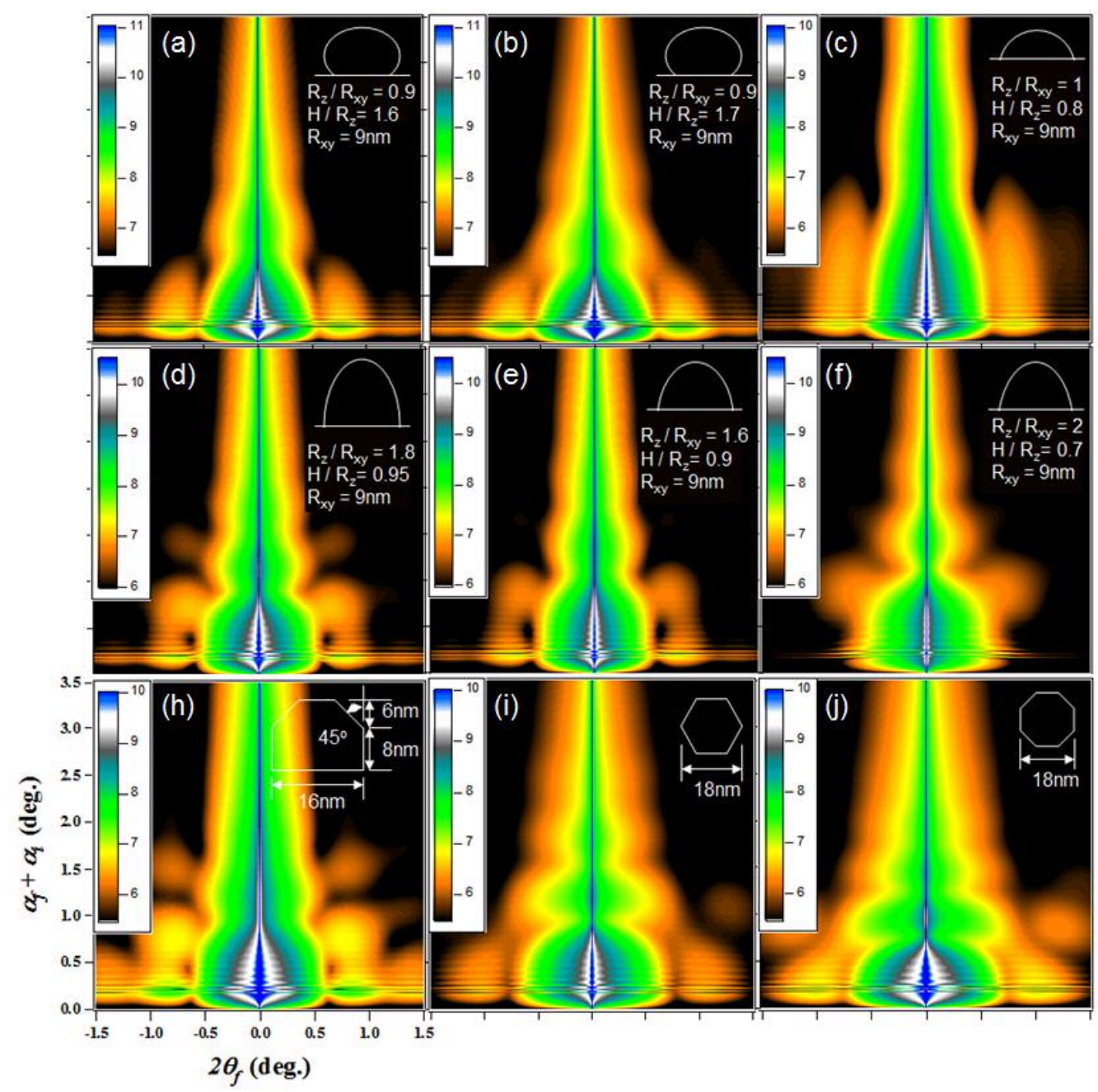

Fig. S1. Simulated GISAXS images for cylinders with various shapes of cross-sections, ellipsoid (a-f) and polygons (h-j). Polydisperse infinitely long cylinder is assumed. X-ray energy of $1.68 \AA$. An incident angle is $0.1^{\circ}$. Polydispersity of the cross-section is $10 \%$. $\mathrm{R}_{\mathrm{xy}}$, $\mathrm{R}_{\mathrm{z}}$, and $\mathrm{H}$ stands for semi-axes of an ellipsoid along the in-plane and z-axis (out-of-plane), and height.

Some cross sections [see Fig. S1(d, e, h)] produce the distinct features in the second lobes of form factor: the blob-like scattering as well as a strong in-plane scattering. As the contact angle at the interface (c and f) is lower than $90^{\circ}$, in-plane scattering gets weaker. On the contrary, as the contact angle higher than $90^{\circ}$, for example hexagon, octagon, and ellipsoids ( $a$ and $b$ ), the in-plane scattering is stronger without making "blob" like features. This is expected because that the scattering predominantly occurs normal to the surface: A round cross-section would make a round scattering pattern. 